\title{
The role of extra-coronary vascular conditions that affect coronary fractional flow reserve estimation.
}

\author{
Jermiah J. Joseph ${ }^{1,2}$, Ting-Yim Lee ${ }^{2}$, Dan Goldman ${ }^{2}$, Christopher W. McIntyre ${ }^{1,2}$, and \\ Sanjay R. Kharche $e^{1,2}$. \\ ${ }^{1}$ Lawson Health Research Institute, London, Canada. \\ ${ }^{2}$ Department of Medical Biophysics, Western University, London, Canada. \\ sanjay.kharche@lhsc.on.ca
}

\begin{abstract}
The treatment of coronary stenosis is often based upon invasive high risk surgical assessment. The surgical assessment quantifies the fractional flow reserve (FFR), a ratio of distal to proximal pressures in respect of the stenosis. Non-invasive imaging-computational methodologies call for robust and calibrated mathematical descriptions of the coronary vasculature that can be personalized. In addition, it is important to understand non-vascular factors that FFR. In this preliminary work, a OD coronary vasculature model capable of personalization was implemented. The model was used to demonstrate the roles of focal and extended stenosis (intra-vascular), as well as microvascular disease and atrial fibrillation (extra-vascular) on FFR. It was found that FFR the right coronary artery is maximally affected by disease conditions. Interestingly, the severity of both microvascular disease and atrial fibrillation were found to be secondary to their mere presence regarding the modelling based FFR estimation. The OD model provides a computationally inexpensive instrument for in silico coronary blood flow investigation as well as clinical-imaging decision making. Furthermore, it establishes a basis for 3D computational fluid dynamics assessment of FFR in patient specific geometries.
\end{abstract}

Keywords: Coronary vasculature, lumped parameter model, fractional flow reserve, computational cardiology.

\section{Introduction}

Coronary artery disease is a major clinical concern, and its diagnosis remains invasive and high risk. The current use of angiographic imaging permits localization of coronary stenosis, but provides minimal utility to generate percutaneous coronary intervention (surgery) recommendations. Fractional flow reserve (FFR) is an increasingly used composite quantity that reflects the severity of stenosis [1]. Clinically, FFR is obtained by using pressure catheters to calculate the ratio of the pressure distal to and proximal to a stenosis that is suitably contrast enhanced, with a value of nil (0) indicating complete stenosis and unity indicating an absence of stenosis. Traditionally, an FFR threshold 0.8 is used to identify clinically significant stenosis in a coronary artery that requires revascularization [2, 3]. However, invasive assessment of FFR is cost prohibitive, in 
addition to presenting high surgical risk to the patient. Surgical and pharmacological sensitivity is increasing relevant where adverse events often occur in critically ill patients, such as those with renal failure $[4,5]$.

In this study, a modelling methodology was developed to assist in computationalimaging FFR assessment. The approach is based on tangible 0D models of human hemodynamics that permit development of cause-effect relationships [6] as well as existing imaging expertise [7]. Circulation models represented by ordinary differential equations (0D models) have been widely used in the literature. We used a previously developed model [8] to uncover causes of pediatric hypertension [6], while others deployed it in the study of atrial fibrillation [9]. A calibrated and biophysically detailed cardiovascular model [10,11] has been widely used. ODE models of organs [12], feedback mechanisms such as baroreflex [ref], and surgical instruments such as dialyzer machines $[13,14]$ have also been used to improve our understanding of processes underlying clinical practices. Others have used composite models to assess the efficacy of surgeries such as dialysis [15].

Recent developments in computational software to develop patient-specific 3D model geometries of vasculature from computerized tomography (CT) and perform hemodynamic blood flow simulations offer an approach to non-invasively assess coronary artery disease [16]. The lumped parameter (0D) approach of modelling cardiovascular systems using the three-element Windkessel representation of vessels has been widely studied to investigate their utility in simulating hemodynamic physiology [17]. By using structured trees to represent small artery bifurcations, outflow boundary conditions can be estimated using 0D models [18, 19]. Coupling 3D finite element models with $0 \mathrm{D}$ and $1 \mathrm{D}$ models of the systemic circulation have been used to investigate their interactions and effects on coronary blood flow simulations [20].

Computationally efficient modelling (0D) for study of coronary blood flow dynamics remains limited [21]. The applicability of 0D models in the personalized assessment of FFR has yet to be undertaken. In this work, we further developed and incorporated an existing model coronary vasculature ODE model [21] and used it to demonstrate important factors that regulate FFR.

\section{$2 \quad$ Methods}

A recent model of the coronary circulation [21] was adapted in this study. The lumped coronary system was further developed, with a detailed heart model [11] including all four chambers (Fig. 1, A). The diameters $\left(D_{n}\right)$ and lengths $\left(l_{n}\right)$ for each vessel were obtained from the literature [21] (Fig. 1, B), and used to assign resistance $\left(\mathrm{R}_{\mathrm{n}}\right)$, compliance $\left(\mathrm{C}_{\mathrm{n}}\right)$, and inductance $\left(\mathrm{L}_{\mathrm{n}}\right)$ values. The microvasculature terminal impedances $\left(Z_{i}\right)$ were estimating using a structured tree model [22], which were used to simulate microvascular disease.

Simulations were designed to explore the effects of stenosis severity with varying a) stenosis length; b) microvascular disease (increase of terminal impedance); and c) atrial fibrillation. Atrial fibrillation (AF) was simulated by reducing both atrial systolic 
elastances from their baseline case ( $0 \% \mathrm{AF})$ to their diastolic values (100\% AF), mimicking the absence of atrial systole. Further, the irregularity in the heartbeat that occurs during AF was simulated by randomly sampling the heart rate from a Gaussian distribution with mean around baseline heart rate and a suitable coefficient of variation, as found in the literature ( $\mu=65 \mathrm{bpm}, \sigma=15.6)$ [9].

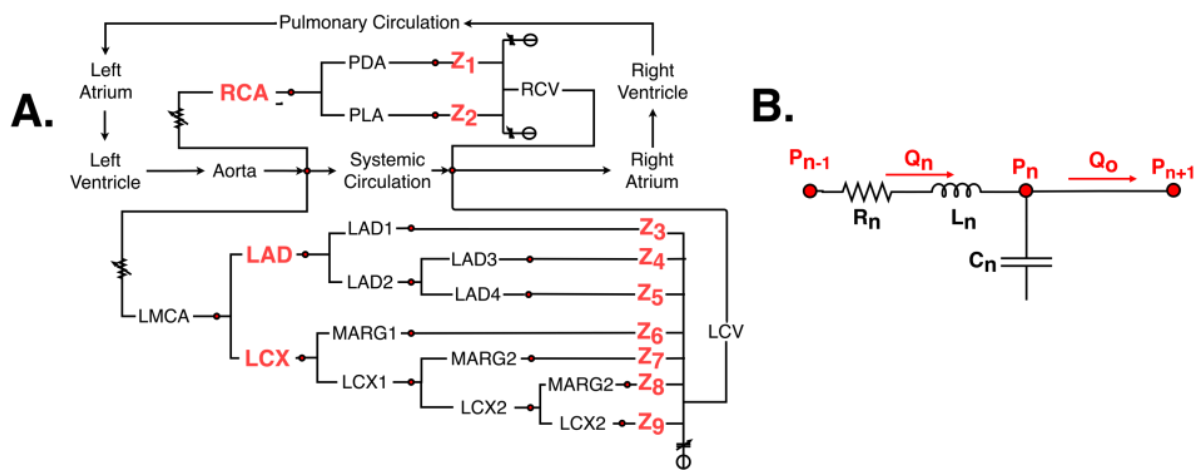

Fig. 1. Electrical analogue of the coronary vasculature model. A: Schematic representation and connectivity coronary vessels. The vessels and impedances in red were used in this study. B: An electrical analog of a blood vessel.

Stenosis in three major vessels, namely the left anterior descending artery (LAD), the left circumflex artery (LCX), and the right coronary artery (RCA), was investigated. Simulations were performed by imposing focal stenosis one of the three vessels or full length stenosis in one of the three vessels. In addition, downstream microvascular disease and atrial fibrillation were imposed. FFR was recorded by calculating the ratio of the pressure distal, $P_{d}$, to the proximal, $P_{a}$, to the stenosis:

$$
F F R=\frac{P_{d}}{P_{a}}
$$

The model is a system of 36 coupled stiff ordinary differential equations. Pressures and flows were computed as state variables. The four chambered heart's elastances generated aortic pressure and flow that provided inlet boundary conditions to the coronary vasculature. The terminal boundaries were considered to remain at constant flow and pressure. The system was solved using our robust solver [6]. The method used in the solver is based on Backward Difference Formulae that provides $O\left(\mathrm{dt}^{6}\right)$ accuracy. A maximum time step of $\mathrm{dt}=0.005 \mathrm{~s}$ gave stable solutions which remained unaffected when the maximum time step was halved.

\section{Results}

Each simulation generated biological activity of $500 \mathrm{~s}$, and the last $2 \mathrm{~s}$ of activity (Fig. 2) were used to generate the results. In the control case (Fig. 2, first row), the FFR values were virtually unity (i.e. more than 0.8 ) for all three coronary vessels. When 
there was a full-length stenosis (Fig. 2, second row; $\alpha=95 \%$ ), the FFR values reduced to 0.56 for the LAD, 0.52 for the LCX, and 0.50 for the RCA. When complete microvascular disease was simulated (Fig. 2, third row), the FFR values were 1 for all three vessels. With atrial fibrillation (Fig. 2, third row; $\mathrm{AF}=75 \%$ ) and intermediate stenosis, the FFR values reduce to $0.9,0.9$, and 0.65 for the LAD, LCX, and RCA, respectively.
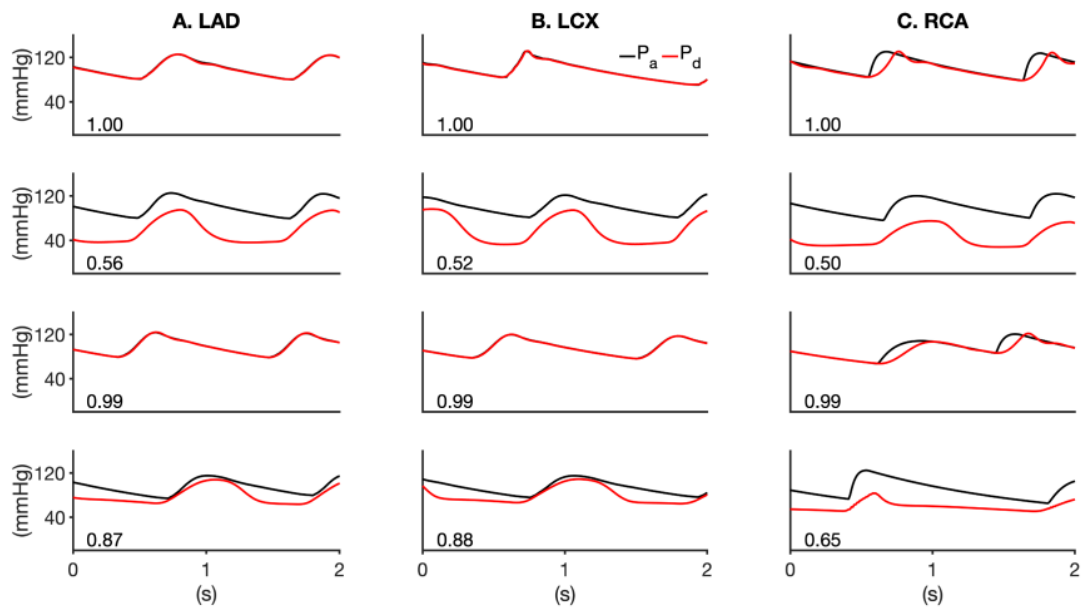

Fig. 2. FFR estimates in the LAD (left column), LCX (middle column), and RCA (right column) under multiple disease conditions. FFR values are provided in each panel. In all panels black lines represent proximal while red lines represent distal pressure. Top row shows non-stenosed model behavior. Second row shows the result of focal stenosis $(\alpha=95 \%)$. The third row shows the result of downstream microvascular disease and no focal stenosis $(\epsilon=2.33)$. Bottom row shows the effect of atrial fibrillation (75\% $\mathrm{AF})$ with intermediate stenosis $(\alpha=50 \%)$.

The dependence of FFR on simultaneous presence of reduced vessel diameters and stenosis length are illustrated in Fig 3. Progressive reduction of RCA diameter was more detrimental as compared to the effects of LAD and LCX diameter reduction. Further, stenosis length devoid of diameter reduction affect the LAD and LCX vasculatures secondary to RCA.
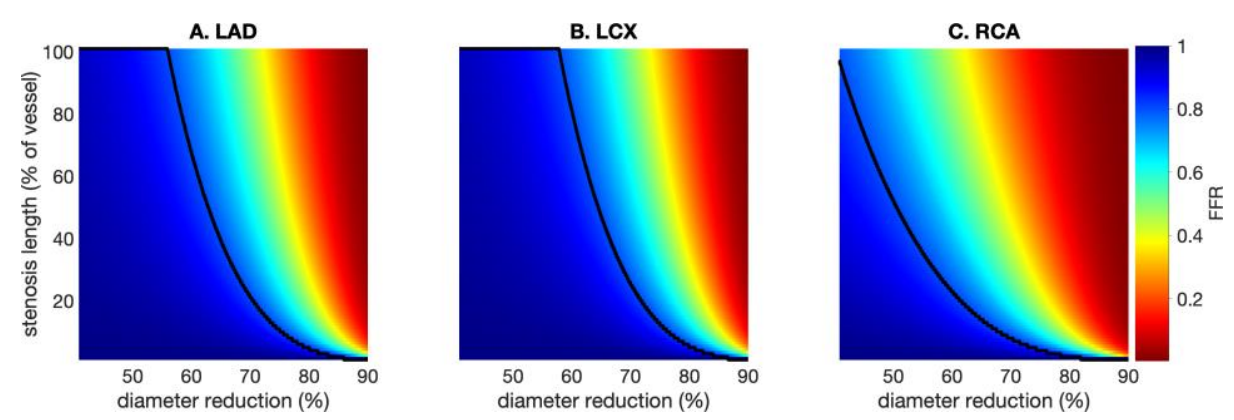
Fig. 3. Dependence of FFR on stenosis length and vessel diameter. The black line represents FFR $=0.8$ threshold value.

The FFR values of simultaneously reducing diameters and imposing varying levels of respective downstream microvascular disease are shown in Fig. 4. An almost unique value of diameter reduction for each, LAD, LCX, and RCA, was observed to characterize FFR transition to below 0.8 in the presence of an arbitrary severity microvascular disease. While the diameter reduction was 0.7 for LAD and LCX, it was seen to be a much lower 0.55 in case of the RCA.
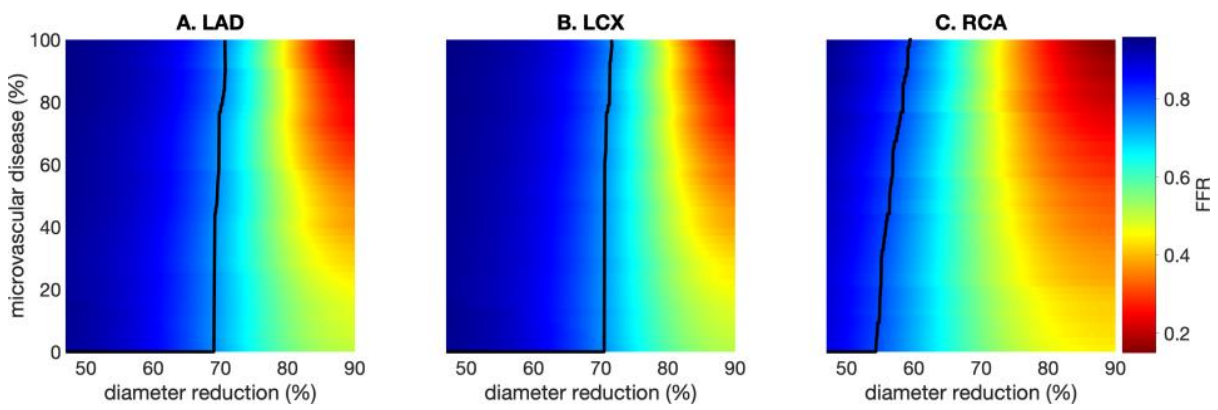

Fig. 4. Dependence of FFR on microvascular disease and vessel diameter. While FFR are color coded as shown in the color bar, the black line represents FFR $=0.8$ threshold. Panels A, B, and C show FFR for LAD, LCX, and RCA, respectively.

The FFR values of simultaneously varying full-length stenosis severity in each vessel and varying levels of AF are shown in Fig. 5. Regardless of the degree of AF severity, a diameter reduction of approximately 0.7 resulted in FFR reducing to under the threshold value. In contrast, a diameter reduction of 0.6 was seen to reduce FFR below threshold values. As is evident in all panels of Fig. 5, FFR estimation also depends on the erratic heart rates.
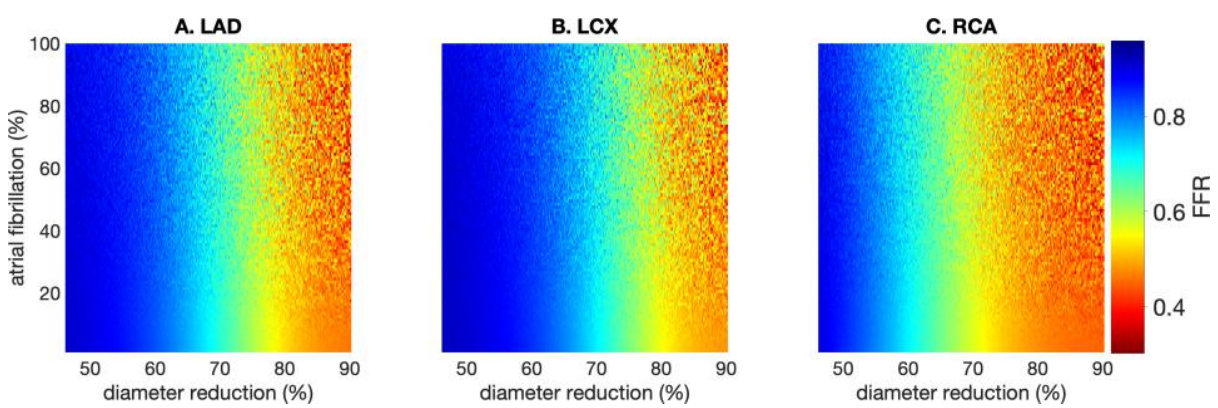

Fig. 5. Dependence of FFR on atrial fibrillation and vessel diameter. FFR values are color coded as shown in the color bar. Panels A, B, and C show FFR data for LAD, LCX, and RCA, respectively. 


\section{Conclusions and Discussion.}

An established lumped parameter model of the human coronary vasculature [21] was further developed in this work (Fig. 1). The model is capable of personalization based on clinical measurements of aortic pressure waves, imaging based vascular geometry (lengths, radii) assessments, as well as cardiac wall motion status [23]. As such, the model permits imaging-clinical data assessment as a computationally efficient instrument, prior to detailed 3D computational fluid dynamics simulations.

Apart from Focal and diffuse coronary stenosis were observed to modulate FFR estimation (Fig. 2). However, our simulations also strongly indicate that FFR estimation must consider other conditions such as $\mathrm{AF}$ and microvascular disease, both of which can be diagnosed non-invasively. Furthermore, it appears that the right ventricle is more susceptible to a reduced blood flow due to the spectrum of conditions, including RCA stenosis (Figs. 3-5). Novel imaging protocols that account for cardiac chamber to chamber diastole will fortify further refinement of the diagnostic instrument. Overall, our 0D model will enhance our future imaging-modelling studies.

\section{Acknowledgements.}

This work was supported by Canarie Inc. (RS-111), Canada Heart and Stroke Foundation grant (G-20-0028717), Canada NSERC operational grant (R4081A03), and NSERC graduate scholarship. We thank Compute Canada for high performance computing resources. We thank Dr. Kapiraj Chandrabalan for editing support.

\section{References}

[1] Fearon, W.F., Tonino, P.A., De Bruyne, B., Siebert, U., Pijls, N.H.: Rationale and design of the Fractional Flow Reserve versus Angiography for Multivessel Evaluation (FAME) study. Am Heart J 154, 632-636 (2007)

[2] Pijls, N.H., van Schaardenburgh, P., Manoharan, G., Boersma, E., Bech, J.W., van't Veer, M., Bar, F., Hoorntje, J., Koolen, J., Wijns, W., de Bruyne, B.: Percutaneous coronary intervention of functionally nonsignificant stenosis: 5-year follow-up of the DEFER Study. J Am Coll Cardiol 49, 2105-2111 (2007)

[3] Tonino, P.A., De Bruyne, B., Pijls, N.H., Siebert, U., Ikeno, F., van' t Veer, M., Klauss, V., Manoharan, G., Engstrom, T., Oldroyd, K.G., Ver Lee, P.N., MacCarthy, P.A., Fearon, W.F.: Fractional flow reserve versus angiography for guiding percutaneous coronary intervention. N Engl J Med 360, 213-224 (2009)

[4] Odudu, A., Francis, S.T., McIntyre, C.W.: MRI for the assessment of organ perfusion in patients with chronic kidney disease. Current opinion in nephrology and hypertension 21, 647-654 (2012)

[5] McIntyre, C.W., Burton, J.O., Selby, N.M., Leccisotti, L., Korsheed, S., Baker, C.S., Camici, P.G.: Hemodialysis-induced cardiac dysfunction is associated with an acute reduction in global and segmental myocardial blood flow. Clinical journal of the American Society of Nephrology : CJASN 3, 19-26 (2008) 
[6] Altamirano-Diaz, L., Kassay, A.D., Serajelahi, B., McIntyre, C.W., Filler, G., Kharche, S.R.: Arterial Hypertension and Unusual Ascending Aortic Dilatation in a Neonate With Acute Kidney Injury: Mechanistic Computer Modeling. Frontiers in Physiology 10, (2019)

[7] Kharche, S.R., So, A., Salerno, F., Lee, T.Y., Ellis, C., Goldman, D., McIntyre, C.W.: Computational Assessment of Blood Flow Heterogeneity in Peritoneal Dialysis Patients' Cardiac Ventricles. Front Physiol 9, 511 (2018)

[8] Shi, Y., Korakianitis, T., Bowles, C.: Numerical simulation of cardiovascular dynamics with different types of VAD assistance. Journal of biomechanics 40, 29192933 (2007)

[9] Anselmino, M., Scarsoglio, S., Saglietto, A., Gaita, F., Ridolfi, L.: A Computational Study on the Relation between Resting Heart Rate and Atrial Fibrillation Hemodynamics under Exercise. PLoS ONE 12, e0169967 (2017)

[10] Heldt, T., Mukkamala, R., Moody, G.B., Mark, R.G.: CVSim: An Open-Source Cardiovascular Simulator for Teaching and Research. The open pacing, electrophysiology \& therapy journal 3, 45-54 (2010)

[11] Heldt, T., Shim, E.B., Kamm, R.D., Mark, R.G.: Computational modeling of cardiovascular response to orthostatic stress. J Appl Physiol (1985) 92, 1239-1254 (2002)

[12] Debbaut, C., Monbaliu, D.R., Segers, P.: Validation and calibration of an electrical analog model of human liver perfusion based on hypothermic machine perfusion experiments. The International journal of artificial organs 37, 486-498 (2014)

[13] Coli, L., Ursino, M., De Pascalis, A., Brighenti, C., Dalmastri, V., La Manna, G., Isola, E., Cianciolo, G., Patrono, D., Boni, P., Stefoni, S.: Evaluation of intradialytic solute and fluid kinetics. Setting Up a predictive mathematical model. Blood Purif 18, $37-49$ (2000)

[14] Ursino, M., Coli, L., Brighenti, C., Chiari, L., de Pascalis, A., Avanzolini, G.: Prediction of solute kinetics, acid-base status, and blood volume changes during profiled hemodialysis. Ann Biomed Eng 28, 204-216 (2000)

[15] Lim, K.M., Choi, S.W., Min, B.G., Shim, E.B.: Numerical Simulation of the Effect of Sodium Profile on Cardiovascular Response to Hemodialysis. Yonsei medical journal 49, 581-591 (2008)

[16] Sankaran, S., Esmaily Moghadam, M., Kahn, A.M., Tseng, E.E., Guccione, J.M., Marsden, A.L.: Patient-specific multiscale modeling of blood flow for coronary artery bypass graft surgery. Ann Biomed Eng 40, 2228-2242 (2012)

[17] Olufsen, M.S., Nadim, A.: On deriving lumped models for blood flow and pressure in the systemic arteries. Mathematical biosciences and engineering : MBE 1, 61-80 (2004)

[18] Olufsen, M.S., Peskin, C.S., Kim, W.Y., Pedersen, E.M., Nadim, A., Larsen, J.: Numerical simulation and experimental validation of blood flow in arteries with structured-tree outflow conditions. Ann Biomed Eng 28, 1281-1299 (2000)

[19] Olufsen, M.S.: A one-dimensional fluid dynamic model of the systemic arteries. Stud Health Technol Inform 71, 79-97 (2000) 
[20] Kim, H.J., Vignon-Clementel, I.E., Coogan, J.S., Figueroa, C.A., Jansen, K.E., Taylor, C.A.: Patient-specific modeling of blood flow and pressure in human coronary arteries. Ann Biomed Eng 38, 3195-3209 (2010)

[21] Duanmu, Z., Yin, M., Fan, X., Yang, X., Luo, X.: A patient-specific lumpedparameter model of coronary circulation. Scientific reports 8, 874 (2018)

[22] Olufsen, M.S.: Structured tree outflow condition for blood flow in larger systemic arteries. Am J Physiol-Heart C 276, H257-H268 (1999)

[23] Crowley, L.E., McIntyre, C.W.: Remote ischaemic conditioning[mdash]therapeutic opportunities in renal medicine. Nature reviews. Nephrology 9, 739-746 (2013) 